\title{
Development of a cervical cancer screening program in a slum setting using visual inspection with acetic acid: Analysis of feasibility and cost
}

\author{
Leslie S. Bradford ${ }^{1}$, Bimalangshu R. Dey², Syed Md Akram Hussain ${ }^{3}$, Sultana Razia Begum ${ }^{3}$, \\ Farhat Hussain $^{3}$, Sharmina Hoque ${ }^{3}$, Annekathryn Goodman ${ }^{1^{*}}$ \\ ${ }^{1}$ Division of Gynecologic Oncology, Massachusetts General Hospital, Boston, USA \\ ${ }^{2}$ Division of Hematologic Oncology, Massachusetts General Hospital, Boston, USA \\ ${ }^{3}$ AK Khan Healthcare Trust, Dhaka, Bangladesh \\ Email: agoodman@partners.org
}

Received 22 April 2012; revised 19 May 2012; accepted 28 May 2012

\begin{abstract}
Objective: In Bangladesh, cervical cancer is the second most common cause of cancer deaths in women. Annually, over 50 million women are at risk for cervical cancer, with 17,686 cases diagnosed and 10,362 deaths each year. Visual inspection with acetic acid (VIA) is a well validated tool in low resource areas for identifying cervical lesions. In this pilot study, AK Khan Health Care Trust (AKKHCT), in collaboration with oncologists at Massachusetts General Hospital (LB, BD, AG), launched a VIA cervical cancerscreening program. Methods: A clinic was established in the Korail Slum of Dhaka, a 90-acre slum with over 70,000 inhabitants, in the summer of 2011. From September 17, 2011 to November 1, 2011, ten women living in the Korail Slum were recruited and completed the three-month training program to learn how to do a pelvic examination and to evaluate the cervix using VIA. Three of the ten women performed VIA screening during the study period. Results: 44 patients were recruited and 8 women were identified to have VIA-positive results for a screen-positive rate of $18.2 \%$. Two of the eight women underwent treatment. One woman underwent an excisional procedure for pre-invasive disease in the slum clinic. The other woman had an early invasive cervical cancer and was successfully treated in a local hospital. Conclusion: Our pilot project demonstrates some important factors in developing and implementing a successful screening program. Involving laywomen as healthcare workers provides a strong tie to community, as well as education, economic independence, and empowerment for women who would otherwise occupy a lower social standing in their community. VIA is fea-
\end{abstract}

"Corresponding author. sible in the complicated environment of an inner city slum. It was feasible to perform minor excisional procedures to treat pre-invasive cervical disease in the slum clinic.

Keywords: Visual Inspection Acetic Acid; VIA; Cervical Cancer; Bangladesh; Dhaka Slum Korail Slum

\section{INTRODUCTION}

Cervical cancer is a disease of poverty and inequity. Infection with high-risk Human Papilloma Virus (HPV) is the major cause of cervical cancer. While HPV infection is common worldwide, the risk of dying from cervical cancer is disproportionately higher among women in low- and middle-income countries [1]. In Bangladesh, cervical cancer is the second most common cause of cancer deaths in women. Annually, over 50 million women are at risk for cervical cancer, with 17,686 cases diagnosed and 10,362 deaths each year. It is projected that by 2025, these numbers will increase to over 31,000 new cases each year and over 18,000 deaths [2]. These deaths often occur in relatively young women, who are raising children, caring for their families, and contributing to the communities.

Cervical cancer is a preventable disease and in developed countries, routine effective screening with cytology (Pap smears) followed by colposcopy for the evaluation of abnormal results, detects the disease at its pre-invasive stage and has reduced the incidence of cervical cancer by almost $80 \%$ [3]. Based on the available data, there are two major challenges facing Bangladesh in regard to the successful prevention of invasive cervical cancer. First, Bangladesh lacks the health care delivery infrastructure to successfully launch large-scale screening programs via cytological approaches. To surmount 
this challenge, alternative screening methods have been recommended for resource-poor women, including visual inspection of the vagina and cervix with acetic acid (VIA) or Lugol's iodine or HPV DNA testing. VIA allows for inspection for suspicious cancerous areas that look "white" after application of dilute acetic acid. Women with a positive VIA can then be triaged directly for colposcopy to establish a conclusive diagnosis and to determine appropriate treatment of pre-invasive disease. Such an approach was recently undertaken in a study in Bangladesh sponsored by United Nations Population Fund (UNFPA), which served to identify the second major challenge that centered on patient noncompliance. In this study, where the model was to screen and then to refer to the designated centers for colposcopy and treatment, there was no effective mechanism to ensure the compliance of the VIA positive women to diagnostic evaluation and management [4].

The current project is a pilot trial targeting the women in the Korail Slum of Dhaka City, Bangladesh, using screening by VIA with immediate colposcopy and treatment, based on a screen-and-treat, rather than screenand-refer approach. The primary goals of this project are to assess the feasibility, safety, and sustainability of such an approach.

\section{MATERIALS AND METHODS}

In this pilot study, AK Khan Health Care Trust (AKKHCT), in collaboration with oncologists at Massachusetts General Hospital (LB, BD, AG), launched a cervical cancer-screening program. Permission to work in Bangladesh was obtained from the Bangladesh Medical and Dental Council. A clinic was established in the Korail Slum of Dhaka, a 90-acre slum with over 70,000 inhabitants, in the summer of 2011. The clinic is equipped with one private examination room for performing VIA, colposcopy and minor procedures (Figure 1).

Electrical outlets, a functioning sink, and an autoclave allow for procedures, such as LEEP (loop electrosurgical excision procedure) and cryotherapy, to be performed in a private setting. All patient data is stored in an electronic health record.

The first step in this program was to recruit local women to undergo a three-week training program in VIA. These women were slum dwellers and had to have, at least, an eight-grade education to ensure literacy. Ten women underwent the training program under the supervision of two senior physicians (SMAH, SRB), which consisted of didactic lectures, practical training, and a final examination (Figure 2). Simultaneously, a general healthcare assessment of the slum was undertaken, using a household survey. Women were invited to partake in cervical cancer screening using VIA through

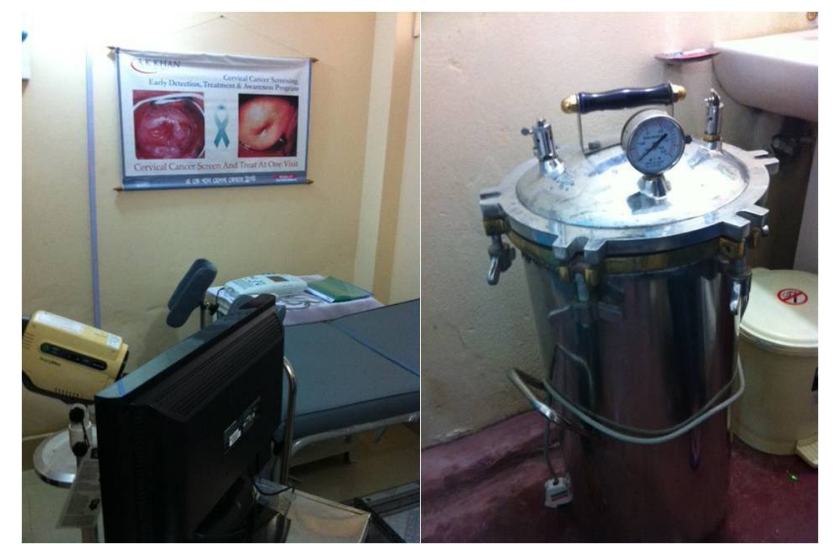

Figure 1. Korial slum clinic, colposcopy, and autoclave.

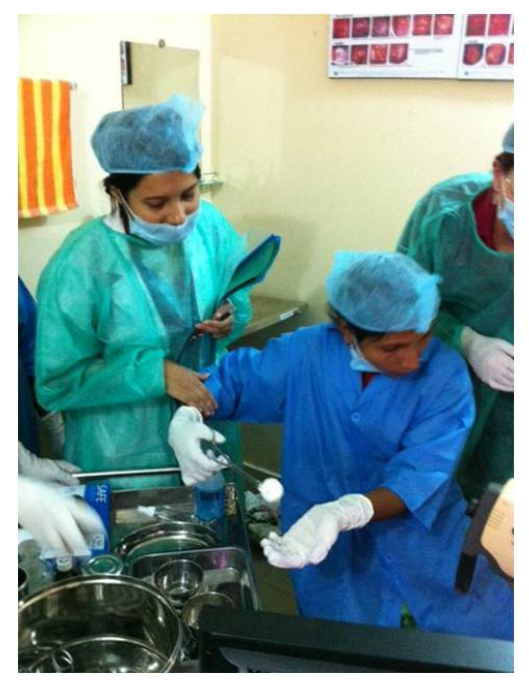

Figure 2. Teaching of VIA to lay healthcare worker in Korail slum clinic.

this project. Specifically, woman ages 20 to 45 years old were recruited. Each patient was offered one screening visit to include medical history, pelvic exam, and VIA.

After counseling and verbal informed consent, VIA was performed beginning with a speculum exam to identify anatomy. Five percent dilute acetic acid was then applied for one minute. VIA positivity was defined by a definite, well-defined acetowhite change noted within the transformation zone in close proximity to the squamocolumnar junction (SCJ). Patients with an unremarkable medical history, exam, and no lesions seen on VIA, were discharged after receiving general health counseling regarding the signs and symptoms of gynecologic cancers (Table 1).

Women with positive VIA screening were referred for colposcopy, performed according to the International Agency on Research on Cancer (IARC) manual on colposcopy [5,6]. Colposcopic findings were recorded in the clinic's electronic health record. Women with normal findings or those consistent with Cervical Intraepithelial 
Table 1. After a normal pelvic examination (negative VIA), women are counseled about the signs of cervical cancer.

\section{SYMPTOMS OF CERVICAL CANCER \\ ABNORMAL BLEEDING \\ POST COITAL BLEEDING \\ ABNORMAL DISCHARGE \\ PELVIC PAIN}

Neoplasia (CIN) 1 or cervicitis were advised to return for repeat examination in 6 months. Women with colposcopic findings of CIN 2 or 3 were offered definitive treatment with LEEP at the time of colposcopy. Specimens were sent for cytology through a local pathology lab at a nearby hospital. Women with findings suggestive of invasive cancer, or who were not deemed safe for treatment in the slum clinic, were referred to a nearby hospital where appropriate treatment, either by LEEP or cold-knife conization, could be performed in an ambulatory surgery setting. A summary of the triage algorithm is summarized in Table 2.

\section{RESULTS}

The project was initiated in June 2011, with preliminary results and costs assessed from the initiation of VIA screening on September 17, 2011 to November 1, 2011. For training laywomen to become healthcare workers and perform VIA, ten women were recruited and completed the three-month training program. Three of the ten women performed VIA screening during the study period. They recruited 44 patients and identified 8 VIA-positive women, for a screen-positive rate of $18.2 \%$. These women were referred for colposcopy where VIA positivity was confirmed by experienced colposcopists.

Of the 8 women undergoing colposcopy, 3 patients were identified as having probable CIN 2 - 3. These women were offered LEEP as part of the see-and-treat program. One woman deferred treatment at the time of diagnosis and was scheduled to return at a later date. Another patient underwent a LEEP, successfully performed in the slum clinic. Final histology confirmed CIN 2 - 3. One patient had a lesion suspicious for carcinoma in situ (CIS) versus invasive carcinoma and was offered cold-knife conization (CKC) at the local hospital. She was subsequently diagnosed with an invasive squamous cell carcinoma of the cervix, Stage IB1. She underwent definitive treatment with a radical hysterectomy and bilateral pelvic lymphadenectomy and has done well postoperatively. These results are summarized in Table 3. The annual projected costs of a full time screening program were projected to be $\$ 87,388$ US dollars. The breakdown of expenses is summarized in Table 4.
Table 2. Treatment algorithm.

WOMEN AGED 20 - 45 RECRUITED

VIA EXAM

IF VIA NEGATIVE THEN GENERAL HEALTH COUNSELING

IF VIA POSITIVE THEN COLPOSCOPY

IF COLPOSCOPY SHOWS CIN 1/CERVICITIS THEN TREAT WITH ANITBIOTIC AND RETURN IN SIX MONTHS

IF COLPOSCOPY SHOWS CIN 1 OR CIN 2 THAEN OFFER LEEP AT THE TIME OF COLPOSCOPY

IF COLPOSCOPY SHOWS CIS OR CANCER THEN REFERRAL TO HOSPITAL FOR CONE BIOPSY OR HYSTERECTOMY

VIA-visual inspection with acteic acid; CIN 1-cervical intraepithelial neoplasia one or mild dysplaisa; CIN 2-cervical intraepithelial neoplasia two or moderate dysplaisa; CIN 3-cervical intraepithelial neoplasia three or severe dysplaisa; CIS—carcinoma-in-situ.

\section{DISCUSSION}

Cervical cancer is a preventable disease because it has a long pre-invasive phase, and there is an effective screening test, the Pap smear. Developed countries have seen a $75 \%$ decrease in incidence and mortality of cervical cancer in the past 50 years because of such screening. In contrast, for low- and middle-income countries, cervical cancer is the second most common cause of cancer-related morbidity and mortality in women: 371,200 new cases annually with a $50 \%$ mortality rate $[7,8]$. These disparities reflect the vast differences between countries and highlight the impact of lack of resources for screening and intervention in low-income countries. In Bangladesh, cervical cancer is the second most common cancer among women of all ages and is leading cause of cancer-related mortality among women [2]. The lack of healthcare infrastructure makes screening with cytology an unattractive model. VIA, on the other hand, is an ideal, low-cost tool for screening in such a resource-poor setting.

The feasibility of VIA has been well documented and validated in other resource poor settings outside of Bangladesh. Within Bangladesh, an initial pilot project launched by Bangabandhu Sheikh Mujib Medical University (BSMMU) and the United Nations Population Fund (UNFPA) performed 11,145 VIA tests in 16 districts as part of the pilot phase. The VIA-positive rate was $4.92 \%$. These women were then referred for colposcopy at BSMMU. The project was later expanded to 22 districts, with a total of 21,609 VIA tests performed, $4.28 \%$ of which were positive. The authors acknowledged that the project required a great deal of resources, both in terms of financial investment and personnel-a total of 347 providers ranging from doctors to nurses to welfare visitors were employed. While most women recruited (84\%) showed interest in screening, and most providers (94\%) were positive about the program, a large pro-portion of providers $(30 \%)$ cited inadequate system infrastructure and clinic space and inadequate numbers of personnel as 
Table 3. Results.

\begin{tabular}{cccccc}
\hline PATIENTS & VIA & COLPOSCOPY & LEEP & CONE & RADICAL HYSTERECTOMY \\
\hline 44 & 44 & 36 & 1 & 1 & 1 \\
Breakdown of patients & & & & & \\
VIA negative & 36 & & & & \\
VIA positive & 8 & 4 & 1 & 1 & 1 \\
CIN 1 & & 3 & & \\
CIN 2/3 & & & & \\
INVASIVE CANCER & & & & & \\
\hline
\end{tabular}

VIA—visual inspection with acteic acid; CIN 1-cervical intraepithelial neoplasia one or mild dysplaisa; CIN 2-cervical intraepithelial neoplasia two or moderate dysplaisa; CIN 3-cervical intraepithelial neoplasia three or severe dysplaisa.

Table 4. Projected annual costs of screening program.

\begin{tabular}{|c|c|c|c|}
\hline CATEGORY & EXPENDITURE & US DOLLARS & $\%$ OF TOTAL \\
\hline \multicolumn{4}{|l|}{ Staff Salaries } \\
\hline & CEO \& Senior Staff & $\$ 48,876$ & $56 \%$ \\
\hline & Other Staff & $\$ 5004$ & $5.8 \%$ \\
\hline \multicolumn{4}{|l|}{ Materials } \\
\hline & Screening Materials & $\$ 2184$ & $2.5 \%$ \\
\hline & Pathology Services & $\$ 1008$ & $1.2 \%$ \\
\hline \multicolumn{4}{|l|}{ Clinic Operations } \\
\hline & Physicians & $\$ 13,980$ & $16 \%$ \\
\hline & Local Health Workers & $\$ 2628$ & $2.8 \%$ \\
\hline & Nursing Staff & $\$ 2328$ & $2.7 \%$ \\
\hline & Clinic Utlilities & $\$ 9100$ & $10.4 \%$ \\
\hline Clinic Maintenance & & $\$ 2280$ & $2.6 \%$ \\
\hline TOTAL COST & & $\$ 87,388$ & \\
\hline
\end{tabular}

obstacles [9]. VIA screening had been provided for 104,098 women across 44 districts in Bangladesh. 5013 (4.8\%) women tested positive and 4371 (87.2\%) underwent colposcopy with sensitivity and specificity of VIA for identifying CIN 2 - 3 were $93.6 \%$ and 58.3\%, respectively. The positive-predictive value was $15.6 \%$. The authors, again, noted the program was unlikely to succeed given the large target population and inadequate infrastructures and organization [4].

While VIA is a well-validated screening tool in resource-poor settings with excellent sensitivity and specificity, the sensitivity of cytology much lower, with reports ranging between $47 \%$ and $62 \%$ [10,11]. Cytology, however, does provide higher specificity compared to VIA. Like cytology, one of the limitations of VIA is that the accuracy of results varies depending on individual interpretation. Initial training and quality control are of utmost importance. This pilot project demonstrates, however, that quality training of lay women can be achieved in a short period of time. Because VIA requires fewer specialized personnel and less infrastructure, training, and equipment, it can be used in more remote or rural locations, as well as less-equipped settings, such as an urban slum.

The benefit of VIA in either of these settings is the immediacy of results and the ability to make clinical management decisions during the same visit, thus decreasing the percentage of women lost to follow-up. In Peru, investigators found that follow-up after cytology screening was poor. In contrast, when a "screen and treat”, or single-visit approach was used, only $9 \%$ of women who screened positive failed to receive treatment 
with the single-visit approach, compared to $44 \%$ of women who were lost to treatment using a multi-visit model [12]. In South Africa, VIA-positive women were randomized to immediate treatment with cyrotherapy versus delayed evaluation. The screen-and-treat approach resulted in a lower prevalence of high-grade cervical cancer precursors [13]. Overall, VIA as part of a see-and-treat approach is effective and is also well-accepted by patients $[6,14]$.

Decreased visits also reduces costs for patients (time away from work, transportation costs, etc.), but can also contribute to improved cost efficacy depending on the screening modality. One study used statistical models to estimate the clinical benefits and cost-effectiveness of various screening tools, including cytology, VIA, and HPV DNA testing [15]. The model suggests that a single lifetime screening visit with testing and immediate treatment for women testing positive, translates to a reduction in the incidence of cervical cancer by $26 \%$ - 32\%. Costs of this approach are less than US \$50 per woman. When comparing various strategies, the authors found that women undergoing a single HPV DNA test at age 35 would cost US \$14 per year of life saved (YLS) and reduce the incidence of cervical cancer by 32 percent, compared to no screening. VIA, in comparison, was the most cost-effective tool and would reduce cervical cancer incidence by 26 percent. Cytology was the least effective and most expensive model [15].

Cryotherapy, a low-cost modality that can be performed by appropriately trained lay people, has been the treatment of choice by many cervical cancer screening projects. The procedure is associated with minimal bleeding, pain, or other complications. Discharge after the procedure is common, but when patients are appropriately counseled, cyrotherapy is generally well-accepted by patients $[16,17]$. However, while most studies routinely counsel women to abstain from sexual activity for at least 4 weeks after the procedure, only $23 \%$ - $49 \%$ are able to do so, and condom use is variable, thus placing these women at higher risk for post-procedure infectious morbidity $[18,19]$. Other disadvantages include treating the entire cervix unnecessarily, risk of inadequate depth of freeze, and risk of long term complications such as cervical stenosis, vaginal scarring or agglutination, hematometria, chronic pelvic pain, or masking of an invasive carcinoma underneath scar.

Loop electrosurgical excision procedure (LEEP), is an alternative treatment modality. Limitations to LEEP include requirements for longer periods of training, medical expertise, as well as practical considerations, such as a reliable source of electricity. LEEP, however, has been shown to be more effective with fewer immediate complications, namely infection, and higher cure rates [20]. The procedure can be done quickly and without pain if lidocaine is employed. Also, lesions can be targeted, thus preserving cervical stroma in women of childbearing age. More importantly, tissue is available for histology should carcinoma in situ (CIS) or invasive carcinoma be suspected. Cure rates with LEEP range from 93.9 - 96.4 for all grades of CIN and are higher that those reported for cyrotherapy (88.3\%) [20-22].

Within Bangladesh, various cervical cancer screening programs have identified some major limitations to implementing such projects, including lack of education and awareness among the general population, a high rate of non-compliance, and an inability to assess the adequacy of the screening program due to a lack of a centralized data-collection system [4].

Albeit on a much smaller scale, this current pilot project demonstrates some important factors in developing and implementing a successful screening program. For one, the electronic health record employed in the clinic allows for long-term monitoring and evaluation. Involving laywomen as healthcare workers provides a strong tie to community, as well as education, economic independence, and empowerment for women who would otherwise occupy a lower social standing in their community. The close proximity of an ambulatory surgical center ensures that patients will not be subjected to a lower quality of care should invasive cancer suspected and histologic diagnosis required. Our experience also demonstrates that excisional procedures using LEEP can be performed safely in a slum setting provided the clinic space is well equipped and providers are well trained.

The screen positive rate of $18.2 \%$ is much higher than rates reported in the literature, which range from $4 \%$ $8 \%$ in similar patient populations $[4,9,23]$. This may reflect the small sample size as well as selection bias, in that women with ongoing gynecologic issues were more likely to present at the time the project was initiated. However such a high screen positive rate may reflect the unique nature of screening in a slum setting and concomitant health conditions. There may be higher rates of cervicitis in this densely populated community in which rates of sexual violence and sexually transmitted infections are thought to be higher, thus resulting in falsepositives with VIA. The small nature of this pilot project has also afforded it with high quality training of the lay healthcare workers. Larger scale programs appear to quickly outgrow their already limited resources, specifically in terms of physician and nursing staff [9,24]. We anticipate that in order to expand the current project to screen a larger area and population of women, a larger workforce will be required in order to maintain quality care.

Working in slum setting presents its own unique set of challenges and obstacles. The majority of studies on cervical cancer screening in resource-poor settings have 
been conducted in less densely populated areas. Within Bangladesh, findings from studies that have highlighted cultural considerations, such as higher success rates with screening programs that employ female providers, are applicable in our setting as well [14]. Rates of non-compliance, however, may be higher due to the itinerant nature of slum dwellers and higher rates of other medical, and namely infectious, comorbidities [25]. A screen-andtreat approach may mitigate noncompliance issues with this unique patient population.

This project has required a substantial investment of resources prior to initiation of the screening program. Obtaining safe clinic space and working with well-trained personnel comprises the largest percentage of cost. Employing lay women to perform VIA greatly reduces operation costs while providing a social benefit to those employed by the program.

In conclusion, our preliminary findings suggest that cervical cancer screening with VIA is safe and feasible in a slum setting. Our experience, thus far, confirms findings from other studies, that there is a general lack of knowledge among women about cervical cancer, its symptoms, and options for screening. We hypothesize that LEEP will be more effective than published cure rates with cryotherapy, but longer follow-up and additional screening will be needed to further assess this. Our program has several strengths, mainly a safe and secure clinical space and a centralized, electronic medical record. The structure of the program and the employment of laywomen from the community will allow us to better assess community perceptions and the effects of poverty on health screening.

\section{REFERENCES}

[1] Sankaranarayanan, R., Bhatla, N., Gravitt, P., Basu, P., Esmy, P.O., Ashrafunnessa, K.S., Ariyaratne, Y., Shah, A. and Nene, B.M. (2008) Human papillomavirus infection and cervical cancer prevention in India, Bangladesh, Sri Lanka, and Nepal. Vaccine, 26S, M43-M52. doi:10.1016/j.vaccine.2008.05.005

[2] WHO/ICO Information Centre on HPV and Cervical Cancer (HPV Information Centre) (2012) Human papillomavirus and related cancers in Kenya. Summary Report 2010. www.who.int/hpvcentre

[3] WHO (1996) Cervical cancer control in developing countries: Memorandum from a WHO meeting. BulletinWorld Health Organ, 74, 345-351.

[4] Nessa, A., Hussain, M.A., Rahman, J.N., Rashid, M.G., Muwonge, R. and Sankaranarayanan, R. (2010) Screening for cervical neoplasia in Bangladesh using visual inspection with acetic acid. International Journal of Gynaecology \& Obstetrics, 111, 115-118.

[5] Sellors, J.W. and Sankaranarayanan, R. (2003) Colposcopy and treatment of cervical intraepithelial neoplasia:
A beginner's manual. IARC Press, Lyon.

[6] Sankaranarayanan, R., Rajkumar, R., Arrossi, S., Theresa, R., Esmy, P.O., Mahé, C., Muwonge, R., Parkin, D.M. and Cherian, J. (2003) Determinants of participation of women in a cervical cancer visual screening trial in rural south India. Cancer Detection and Prevention, 27, 457465. doi:10.1016/j.cdp.2003.09.006

[7] Ferlay, J., Shin, H.R., Bray, F., Forman, D., Mathers, C. and Parkin, D.M. (2010). Estimates of worldwide burden of cancer in 2008: GLOBOCAN 2008. International Journal of Cancer, 127, 2893-2917. doi:10.1002/ijc.25516

[8] Jemal, A., Bray, F., Center, M.M., Ferlay, J., Ward, E. and Forman, D. (2011) Global cancer statistics. CA Cancer Journal for Clinicians, 61, 69-90. doi:10.3322/caac.20107

[9] Ahmed, T., Ashrafunnessa and Rahman, J. (2008) Development of a visual inspection programme for cervical cancer prevention in Bangladesh. Reproductive Health Matters, 16, 78-85. doi:10.1016/S0968-8080(08)32419-7

[10] Sankaranarayanan, R., Gaffikin, L., Jacob, M., Sellors, J. and Robles, S. (2005) A critical assessment of screening methods for cervical neoplasia. International Journal of Gynaecology and Obstetrics, 89, S4-S12. doi:10.1016/j.ijgo.2005.01.009

[11] Arbyn, M., Sankaranarayanan, R., Muwonge, R., Keita N., Dolo, A., Mbalawa, C.G., Nouhou, H., Sakande, B., Wesley, R., Somanathan, T., Sharma, A., Shastri, S. and Basu, P. (2008) Pooled analysis of the accuracy of five cervical cancer screening tests assessed in eleven studies in Africa and India. International Journal of Cancer, 123, 153-160. doi:10.1002/ijc.23489

[12] Luciani, S. and Winkler, J. (2006) Cervical cancer prevention in Peru: Lessons learned from the TATI demonstration project. Pan American Health Organization, Washington DC.

[13] Denny, L., Kuhn, L., De Souza, M., Pollack, A.E., Dupree, W. and Wright, T.C. (2005) Screen-and-treat approaches for cervical cancer prevention in low-resource settings: A randomized controlled trial. The Journal of the American Medical Association, 294, 2173-2181. doi:10.1001/jama.294.17.2173

[14] Ansink, A.C., Tolhurst, R., Haque, R., Saha, S., Datta, S. and van den Broek, N.R. (2008) Cervical cancer screening in Bangladesh: Community perceptions of cervical cancer and cervical cancer screening. Transactions of the Royal Society of Tropical Medicine and Hygiene, 102, 499505. doi:10.1016/j.trstmh.2008.01.022

[15] Goldie, S.J., Kuhn, L., Denny, L., Pollack, A. and Wright, T.C. (2001) Policy analysis of cervical cancer screening strategies in low-resource settings: Clinical benefits and cost-effectiveness. The Journal of the American Medical Association, 285, 3107-3115. doi:10.1001/jama.285.24.3107

[16] Alliance for Cervical Cancer Prevention (ACCP) (2003) Effectiveness, safety, and acceptability of cryotherapy. A Systematic Literature Review, ACCP, Seattle.

[17] Jacob, M., Broekhuizen, F.F., Castro, W. and Sellors, J. (2005) Experience using cryotherapy for treatment of cervical precancerous lesions in low-resource settings. 
International Journal of Gynaecology \& Obstetrics, 89, S13-S20. doi:10.1016/j.ijgo.2005.01.026

[18] Alliance for Cervical Cancer Prevention (2004) Improving screening coverage rates of cervical cancer prevention programs: A focus on communities. ACCP, Seattle.

[19] Bingham, A., Bishop, A., Coffey, P., Winkler, J., Bradley, J., Dzuba, I. and Agurto, I. (2003) Factors affecting utilization of cervical cancer prevention services in low-resource settings. Salud Publica de Mexico, 45, S408-S416. doi:10.1590/S0036-36342003000900015

[20] Chirenje, Z.M., Rusakaniko, S., Akino, V. and Mlingo, M. (2001) A randomised clinical trial of loop electrosurgical excision procedure (LEEP) versus cryotherapy in the treatment of cervical intraepithelial neoplasia. American Journal of Obstetrics \& Gynaecology, 21, 617-621. doi:10.1080/01443610120085618

[21] Sankaranarayanan, R., Keshkar, V., Kothari, A., Kane, S., Fayette, J.M. and Shastri, S. (2009) Effectiveness and safety of loop electrosurgical excision procedure for cervical neoplasia in rural India. International Journal of Gynaecology \& Obstetrics, 104, 95-99.

\section{doi:10.1016/j.ijgo.2008.09.009}

[22] Rema, P., Suchetha, S., Thara, S., Fayette, J.M., Wesley, R. and Sankaranarayanan, R. (2008) Effectiveness and safety of loop electrosurgical excision procedure in a lowresource setting. International Journal of Gynaecology \& Obstetrics, 103, 105-110. doi:10.1016/j.ijgo.2008.06.020

[23] Abdel-Hady, E.S., Emam, M., Al-Gohary, A., Hassan, M., Farag, M.K. and Abo-Elkheir, M. (2006) Screening for cervical carcinoma using visual inspection with acetic acid. International Journal of Gynaecology \& Obstetrics, 93, 118-122. doi:10.1016/j.ijgo.2006.01.024

[24] Basu, P., Nessa, A., Majid, M., Rahman, J.N. and Ahmed, T. (2010) Evaluation of the national cervical cancer screening programme of Bangladesh and the formulation of quality assurance guidelines. Journal of Family Planning and Reproductive Health Care, 36, 131-134. doi:10.1783/147118910791749218

[25] Sclar, E.D., Garau, P. and Carolini, G. (2005) The 21st century health challenge of slums and cities. Lancet, 365, 901-903. doi:10.1016/S0140-6736(05)71049-7 